\title{
A28807, Rat Strain
}

National Cancer Institute

\section{Source}

National Cancer Institute. A28807, Rat Strain. NCI Thesaurus. Code C14393.

Very docile. Susceptible to the induction of autoimmune thyroiditis. Low and intermediate antibody responses to 1 and $10 \mathrm{~g}$ BSA, respectively. Low antibody response to streptococcal group A carbohydrate; not linked to the MHC. High relative heart weight in 10-week old males (20/23). 